\title{
TITLE:
}

\section{Mapping the Ethical Issues of Brain Organoid Research and Application}

\section{$\operatorname{AUTHOR}(S)$ :}

Sawai, Tsutomu; Hayashi, Yoshiyuki; Niikawa, Takuya; Shepherd, Joshua; Thomas, Elizabeth; Lee, Tsung-Ling; Erler, Alexandre; Watanabe, Momoko; Sakaguchi, Hideya

\section{CITATION:}

Sawai, Tsutomu ... [et al]. Mapping the Ethical Issues of Brain Organoid Research and Application. AJOB Neuroscience 2021

\section{ISSUE DATE:}

2021-03-26

URL:

http://hdl.handle.net/2433/262700

\section{RIGHT:}

This is an Accepted Manuscript of an article published by Taylor \& Francis in 'AJOB Neuroscience' on 2021, available online: https://www.tandfonline.com/10.1080/21507740.2021.1896603; The full-text file will be made open to the public on 26 March 2022 in accordance with publisher's 'Terms and Conditions for Self-Archiving'.; This is not the published version. Please cite only the published version.; この論文は出版社版でありません。引用の際には出版社版をご確認ご 利用ください。 
FINAL DRAFT: 2021 in AJOB Neuroscience

\section{Mapping the Ethical Issues of Brain Organoid Research and Application}

Tsutomu Sawai ${ }^{1,2 * \dagger}$, Yoshiyuki Hayashi ${ }^{3 \dagger}$, Takuya Niikawa ${ }^{4}$, Joshua Shepherd ${ }^{5,6}$, Elizabeth Thomas ${ }^{7}$, Tsung-Ling Lee ${ }^{8}$, Alexandre Erler ${ }^{9}$, Momoko Watanabe ${ }^{10}$, Hideya Sakaguchi ${ }^{11}$

1) Institute for the Advanced Study of Human Biology (WPI-ASHBi), KUIAS Kyoto University, Japan

2) Uehiro Research Division for iPS Cell Ethics, Center for iPS Cell Research and Application, Kyoto University, Japan

3) Faculty of Medicine, Saitama Medical University, Japan

4) Graduate School of Humanities, Kobe University, Japan

5) Department of Philosophy, Carleton University, Canda

6) University of Barcelona, Spain

7) University of North Carolina School of Medicine, Chapel Hill, North Carolina, USA

8) Graduate Institute of Health and Biotechnology of Law, Taiwan Medical University, China

9) Department of Philosophy and CUHK Centre for Bioethics, The Chinese University of Hong Kong, China

10) Department of Anatomy and Neurobiology, Sue and Bill Gross Stem Cell Research Center, School of Medicine, University of California, Irvine, USA

11) RIKEN Center for Biosystems Dynamics Research, RIKEN BDR-Otsuka Pharmaceutical Collaboration Center, Japan

* Corresponding author: Dr Tsutomu Sawai (sawai.tsutomu.4s@kyoto-u.ac.jp)

$\uparrow$ Equal contribution

\section{Funding}

TS was funded by the JSPS KAKENHI Grant-in-Aid for Young Scientists (B) (Grant Number: 17K13843) and an ASHBi Fusion Research Grant. JS was funded by the ERC Starting Grant (Grant Number: 757698), and an Azrieli Global Scholar fellowship from CIFAR. 
FINAL DRAFT: 2021 in AJOB Neuroscience

\section{Introduction}

Organoid technology has been rapidly advancing in recent years. "Organoids" are three-dimensional structures created by imitating the process of organ formation in vitro using pluripotent stem cells, such as induced pluripotent stem cells (iPSCs) and embryonic stem cells (ESCs). In 2008, researchers created human three-dimensional neural tissue - known as the pioneering work of "brain organoids." In recent years, some researchers have transplanted human brain organoids into animal brains for applicational purposes. With these experiments have come many ethical concerns. It is thus an urgent task to clarify what is ethically permissible and impermissible in brain organoid research. Delayed consideration of ethical issues may unnecessarily fuel public unrest and adversely affect current basic research.

Although multiple academic groups have examined the ethical issues of brain organoid research (Farahany, et al. 2018, Koplin and Savulescu 2019b, Hyun, et al. 2020, Lavazza 2020), the issues have not yet been comprehensively identified and tackled. As such, this paper seeks (1) to sort out the ethical issues related to brain organoid research and application and (2) to propose future directions for additional ethical consideration and policy debates in the field. Toward (1), this paper first outlines the current state of brain organoid research, and then briefly responds to previously raised related ethical concerns. Looking next at anticipated scientific developments in brain organoid research, we will discuss i) ethical issues related to in vitro brain organoids, ii) ethical issues raised when brain organoids form complexes or have relationships with other entities, and iii) ethical issues of research ethics and governance. Finally, in pursuit of (2), we propose research policies that are mindful of the ethics of brain organoid research and application and also suggest the need for an international framework for research and application of brain organoids.

\section{Brain Organoid Research: State of the Science and Ethical Concerns}

State of the Science

In 2008, Yoshiki Sasai and his colleagues generated three-dimensional (3D) 
FINAL DRAFT: 2021 in AJOB Neuroscience

cerebral tissue from mouse and human ESCs (Eiraku, et al. 2008). In this study, the induced mouse cerebral tissue showed synchronized neural function and was successfully transplanted and integrated into mouse brains. Also, the cerebral tissues responded to extrinsic signals to regionalize different domains of the telencephalon. The same group reported a follow-up study that demonstrated improved cerebral tissue that recapitulates some human specific features in 2013 (Kadoshima, et al. 2013).

In the same year, Jürgen Knoblich, Madeline Lancaster, and their colleagues also produced 3D human brain tissue (Lancaster, et al. 2013). The group dubbed their structures "cerebral organoids," for tissues mimicking the developing cerebrum. So far, various region-specific brain organoids have been generated, including the cerebral cortex, hypothalamus, ventral telencephalon, optic cup, anterior pituitary, cerebellum, hippocampus, choroid plexus, and thalamus (Wataya, et al. 2008, Danjo, et al. 2011, Eiraku, et al. 2011, Suga, et al. 2011, Nakano, et al. 2012, Nasu, et al. 2012, Watanabe, et al. 2012, Kadoshima, et al. 2013, Kuwahara, et al. 2015, Muguruma, et al. 2015, Sakaguchi, et al. 2015, Hasegawa, et al. 2016, Ishida, et al. 2016, Ozone, et al. 2016, Shiraishi, et al. 2017, Takata, et al. 2017).

Brain organoids provide unprecedented opportunities to study human neurodevelopment and disease, and can be a useful platform to screen drugs. Commonly used animal models have been very useful, but there are human specific features that cannot be recapitulated in lower species. Human monolayer cell culture has been one approach; however, it cannot fully mimic 3D architecture, which is likely important for predicting clinical outcomes and finding effective drugs. Thus, organoids are of particular strength by providing accessible human 3D brain-like tissues that would provide a better platform to study neurological disorders.

Zika virus outbreak demonstrated an early example of brain organoids as a powerful tool for disease modeling. In 2015, Zika virus infection during pregnancy led to birth defects including microcephaly in South America. Concern of the virus spreading to North America drove a great deal of attention from the American scientific community. Amidst this backdrop, a series of studies used cerebral organoids to elucidate the cause of microcephaly due to Zika virus infection, create disease models, and identify 
FINAL DRAFT: 2021 in AJOB Neuroscience

candidates for therapeutic agents such as TLR3 inhibitor, Ivermectin, and Duramycine (Cugola, et al. 2016, Dang, et al. 2016, Garcez, et al. 2016, Qian, et al. 2016, Watanabe, et al. 2017).

Despite the clear utility of brain organoids, many challenges remain including the incomplete recapitulation of brain structure, size, maturity, vascularization, lamination and lack of input/output systems, external stimuli, and non-neuroectodermal cells. Currently, researchers are trying to tackle these challenges by comparing brain organoids to human fetal tissues to decipher the lack of developmental maturity and diversity (Pollen, et al. 2019, Bhaduri, et al. 2020), transplanting brain organoids into animal brains for the host vascularization (Mansour, et al., 2018), slicing organoids for better layered-organization (Qian, et al. 2020), fusing organoids or spheroids (that is, aggregated neural tissues) to make input/output systems (Xiang, et al. 2019), and adding non-neuroectodermal cells such as microglia and oligodendrocyte to create more complete cerebral tissues (Madhavan, et al. 2018, Ormel, et al. 2018). These approaches have been under development and many scientists are aiming to enhance the cerebral organoids to fully recapitulate the developing human brain for better disease modeling.

\section{Ethical Concerns Raised by Current Brain Organoid Research}

The ethical questions raised by previous studies can be categorized into the following topics; 1) in case that cerebral organoids have interacted with other tissues, 2) in case that cerebral organoids have become integrated with an in vivo brain, and 3) whether or not organoids have consciousness by themselves in vitro.

For the topic 1), one paper reported brain organoids that possessed direct synaptic connections between cerebral neurons and photoreceptor cells, which responded to external light stimuli (Quadrato, et al. 2017). However, the direct connection between cerebral neurons and photoreceptor cells is different from the in vivo visual pathway. In vivo, the photoreceptor cells connect to the optic nerve, which is sent to the bilateral thalamus through electrical and chemical signals and then to the visual cortex (fields 17 and 18) of the cerebrum to be perceived as information in the visual field. Here, all of the distribution of photoreceptor cells, the relay of the thalamus, and the area of the cerebrum 
FINAL DRAFT: 2021 in AJOB Neuroscience

to recognize the given light information as the visual field are required.

It is also believed that activity in the visual field is combined with past memories by comparing it to memories stored in other areas of the cerebrum and the processing of sensory experience is very complex. Thus, the direct connection between cerebral neurons and photoreceptor cells does not necessarily mean the generation of brain-like tissues with vision. The current study is far away from the actual recapitulation of the complex visual system in vivo. However, in the future, due to the progress of scientific technologies like assembloids (see, for instance, the recent report of the assembly of a fused cortical-spinal-muscle organoid [Andersen et al. 2020]), it will be possible to generate the fusion of cerebral, thalamic, and retinal organoids. This would enable the construction of the full visual pathway and might potentially open ethical concerns related to an entity capable of visual perception and pain in vitro.

Regarding the topic 2), the series of transplantation studies of cerebral organoids into the animal brain brought the ethical concerns regarding the creation of human-animal chimeras. One study showed vascularization of transplanted cerebral organoid from host brain and the function of transplanted organoids (Mansour et al. 2018). Another study showed transplanted organoids provided better graft survival with neuronal differentiation compared with the transplantation of neural progenitor cells derived from human PSCs (Daviaud, et al. 2018). These studies did not focus on the connection between the host and the graft via appropriate neural circuits. However, a very recent paper showed the axonal extension from organoids transplanted in the motor area to the spinal cord via the cortico-spinal tract which is a major motor related circuit in the brain (Kitahara, et al. 2020).

Once the appropriate neural circuit is formed from transplanted organoids, the host with chimeric brain might have functional contribution from transplanted human brain organoids, possibly leading to enhanced and acquired ability that does not exist originally. Furthermore, transplanted organoids could disturb the host brain activity via unnatural activation or inhibition. This kind of topic has similarity with the ethical concern regarding the creation of chimeric animal brain by blastocyst complementation. Thus, the existing model might have already created a chimeric brain that has aberrant 
FINAL DRAFT: 2021 in AJOB Neuroscience

cognitive function, which should be carefully characterized scientifically and brought to ethicists' attention for future consideration.

Regarding the topic 3), the studies that revealed brain organoid activities have opened the possibility of the generation of consciousness in vitro. Some recent studies have focused on detecting complex neural activity consisting of synchronized oscillation with individual firing in neural networks (Sakaguchi, et al. 2019), reproducing a waveform from in vitro human brain organoids similar to those seen in preterm neonatal electroencephalography (EEG) (Trujillo, et al. 2019), and demonstrating epileptiform network activity (Samarasinghe, et al. 2019). On some theories of consciousness, such as integrated information theory (IIT) - which postulates that consciousness amounts to the integration of a certain amount of information by some system (Tononi 2012, Tononi, et al. 2016) - this may seem sufficient for attributing consciousness to an in vitro organoid.

All of the complex neural activities in Sakaguchi's work, neonatal EEG-like activity in Muotri's work, and epilepsy-like neural activity in Novitch's group can correspond to information in IIT. These data do not directly mean that these activities bring consciousness because they lack integration of the activity with the surrounding environment. Based on IIT, however, they might have consciousness even if they have no connections with some materials that bind the organoid with the outer world. Thus, it is possible to say that these studies involve the ethical issue of the generation of consciousness in vitro, though we have to pay attention to the fact that IIT is not fully accepted in the scientific community.

Even with these more cautionary views of the progress claimed by recent brain organoid studies, it is undeniable that the field has made tremendous progress. This scientific advancement demands ethical reasoning and guidance, the subject of the rest of this paper.

\section{Ethical Issues Associated with Brain Organoid Research and Application}

Given the rapid rate of progress in brain organoid research, it is prudent to consider not only existing but also anticipated future ethical issues (indeed, some previous 
FINAL DRAFT: 2021 in AJOB Neuroscience

ethical studies have examined existing and potential issues (Lavazza and Massimini 2018, Hostiuc, et al. 2019, Koplin and Savulescu 2019b, Sawai, et al. 2019, Lavazza 2020)). Currently, human brain organoids have been produced and used in vitro, transplanted into rodents, and transplanted into rhesus monkeys (Kitahara, et al. 2020). Though animal transplantation experiments have thus far been conducted as basic research, future preclinical studies may evaluate therapeutic organoid transplantation in humans (Chen, et al. 2019a, 2019b). In light of the current and future state of the field, the following sections will discuss 1) issues raised by the in vitro brain organoid itself, 2) issues raised by transplanting brain organoids into the brains of living organisms or connection with non-living ones, and 3) issues of research ethics and governance, with an eye toward suggestions for future brain organoid research.

\section{Ethical Issues raised by In Vitro Brain Organoids}

If researchers continue developing brain organoids in structure, size, cellular complexity and maturity, brain organoids cultured in vitro may have some morally relevant consciousness. This possibility raises questions regarding brain organoids' moral status. This section delineates related issues, focusing primarily on concerns about consciousness. After introducing the notion of consciousness, we discuss the problems of (i) detection of consciousness, (ii) moral relevance of consciousness, (iii) functional role of bodies, and (iv) moral relevance of self-consciousness.

(i) Detection of consciousness

Consciousness could have profound practical implications for brain organoid research. Although there are many distinctions among the numerous kinds of consciousness (Van Gulick 2018), this paper (and others so far) mainly focuses on phenomenal consciousness which is characterized by what-it's-likeness (Nagel 1974), or what it is like to be in such-and-such mental states. This means if one has phenomenally conscious mental states, he or she experiences (and sometimes enjoys) certain "feels" that accompany them. Typical examples of such feels include the painfulness of pain or bitterness of tasting coffee. We focus on phenomenal consciousness, and particularly on 
FINAL DRAFT: 2021 in AJOB Neuroscience

conscious states with hedonic valence, because it seems morally relevant to have experiences like pain. Phenomenal consciousness has also been the source of the "hard problem" of consciousness in the philosophy of mind for decades (Chalmers 1995).

We could divide the problem of consciousness in brain organoids roughly into two stages. Firstly, we need to know how to detect if the organoids are phenomenally conscious. Secondly, after a successful detection of consciousness, we need to see exactly how the presence of consciousness would be morally relevant. Below, we will discuss these two stages in turn.

The detection of consciousness in brain organoids poses difficult empirical and epistemological problems. In current scientific practice, the gold standard for evidence of consciousness is an introspective report. This measure is hardly foolproof, and is arguably only applicable to humans with linguistic abilities. This concern might suggest the method that Davies and Levy call the silver standard for consciousness detection - the search for evidence of intentional agency (or action) guided by the brain or entity in question (Davies and Levy 2016). Applying this method has suggested that in some cases, humans diagnosed as in 'persistent vegetative states' due to traumatic brain injuries may actually retain consciousness (Owen, et al. 2006, Boly, et al. 2007, Monti, et al. 2010, Cruse, et al. 2012, Fernandez-Espejo and Owen 2013, Owen 2019) with the exhibition of both. As the evidence of intentional agency methodology asks patients to imagine certain scenarios, its results arguably only apply to humans with linguistic comprehension abilities. Some different methodologies for detecting intentional agency might be envisioned for application to brain organoids, but we are not aware of any plausible candidates at the present time.

A different, non-introspective method may hold some promise for brain organoid application. Lavazza and Massimini (2018) propose a promising method for consciousness detection by using an existing measurement method called the Perturbational Complexity Index (PCI). The PCI has theoretical connections to IIT (Lavazza and Massimini 2018). IIT is controversial (Bayne 2018), but one does not need to accept it to see the value in the PCI. It is a measure of the complexity of the response of the thalamocortical system to direct perturbation (by transcranial magnetic stimulation) 
FINAL DRAFT: 2021 in AJOB Neuroscience

(Casali, et al. 2013).

The PCI is better than chance at discriminating between levels of consciousness in humans. That is, the PCI can discriminate between [a] wakefulness and NREM sleep in healthy adults, [b] different levels of sedation with propofol anesthesia, and [c] different conditions following traumatic brain injury. However, even if the PCI is a good candidate for a biomarker of consciousness in well-developed human brains, the measure would not work in very different neural structures such as a brain organoid. At this point, the PCI might be best seen as proof of principle that biomarkers for consciousness can be developed and relied upon as evidence for consciousness. Application of PCI evaluation for organoid study looks unfeasible because the method of transcranial magnetic stimulation (TMS) modulation with EEG recording needs the size and complexity of a whole brain which is lacking in current organoids. But once the complex neural circuits with much space or micro-equipment of PCI for organoids are achieved, the evaluation of PCI for organoids could be performed. We may require more knowledge about both the architecture of different organoids and what kinds of signals qualify as evidence of consciousness in normal brains before the novel application of an existing biomarker could be justified.

(ii) Moral relevance of consciousness

Generally speaking, even if it is uncertain whether an entity has consciousness, it might be the case that we must take certain moral attitudes or actions towards them, whatever such attitudes and actions are. Under such circumstances, it would be wise to choose what to do more conservatively, namely, in a precautionary way that would exclude false negatives. Hence, it would be prudent to proceed under the assumption that the entity has consciousness for the time being by applying a precautionary principle (Birch 2017, Shepherd forthcoming). As Birch has it, "[i]n broad terms, the idea is clearly that we should not require absolute certainty that a species is sentient before affording it a degree of legal protection. Absolute certainty will never be attained (indeed, the "problem of other minds" suggests it cannot even be attained with respect to human minds), and its absence is not a good reason to deny basic legal protections to potentially 
FINAL DRAFT: 2021 in AJOB Neuroscience

sentient animals" (Birch 2017: 2).

Carefully deciding how exactly to apply a precautionary principle to possibly conscious entities will likely require significant collaboration between scientists and policy-makers. But the basic idea seems wise. Deciding which of the many competing theories of phenomenal consciousness is the winner among all will not be possible in the near future. Although some influential theories including IIT and Global Neural Workspace Theory (GWT) (Dehaene and Naccache 2001) might be applicable to brain organoids (Lavazza 2020) for now it is uncertain whether these theories would fully unveil the nature of consciousness. Instead, some have advocated that we search for consensus on common features shared by each theory (Shepherd 2018, Wiese 2020). Assuming that brain organoids themselves may have some consciousness, some of the authors of this paper have already started phenomenological consideration of what kind of consciousness they may have and what value consciousness has.

Despite the problems of detection, we need to know how consciousness in the organoids would be morally relevant. Recently, phenomenal consciousness has not only been of purely philosophical interest but of practical ethics interest (Kahane and Savulescu 2009) . The capacity for phenomenal consciousness is often regarded as equivalent to sentience, which is the ability to feel pain, pleasure, and distress. Given this understanding of phenomenal consciousness, some argue that possessing phenomenal consciousness is thus sufficient to underwrite moral status (Levy and Savulescu 2009), possession of which would entitle an entity to morally appropriate treatment. Yet, this explanation may not be quite right. Some draw a distinction between conscious experiences that have a valence (that are good or bad for the subject) and those that do not (Shepherd 2018, Lee 2019). One moral view is that only experiences with valence have moral value, and thus undergird moral status. Another moral view is that any conscious experience has moral value, and so undergirds moral status. This distinction is relevant to organoids, where one can imagine an organoid with a well-developed visual cortex (say) but no pain system.

Although clearly, phenomenal consciousness is the basis of moral consideration, it is not immediately apparent that creating an entity with such consciousness for research 
FINAL DRAFT: 2021 in AJOB Neuroscience

use should be prohibited (Koplin and Savulescu 2019b). Indeed, common research animals such as rodents and non-human primates are usually taken to have some conscious experiences (although there is active discussion around the ethics of animal experiments (Visak and Garner 2016, Beauchamp and DeGrazia 2020). See also (b) below.).

In addition to the problem of detection and moral relevance, we suggest further problems concerning organoids' consciousness and cognition and their relationships with morality.

(iii) Functional role of bodies

We need to consider the functional role of bodies potentially connected to brain organoids. Being disembodied, brain organoids may constitute "islands of awareness" streams or systems of consciousness unshaped by sensory input and unable to produce expressions via motor output (Bayne, et al. 2020). Current brain organoids may be in a peculiar or even unique state of consciousness because they have no body, sensory input, or motor output. That said, brain organoids of the future may be given "bodies" by such means as programming using artificial gene circuits, connection with animals/humans, connection with other organs of biological origin, or connection with non-living materials (Munsie, et al. 2017, Hyun, et al. 2020). Consequently, future brain organoids may have a different state of consciousness with bodies. There is ongoing active exploration regarding what kind of consciousness developmentally different or embodied brain organoids may have, and the moral implications of these various kinds of consciousness.

(iv) Moral relevance of self-consciousness

We also need to consider the moral relevance of more advanced cognitive abilities other than phenomenal consciousness. While most authors discussing this issue agree that sentience is sufficient for ethical consideration, they also generally hold that self-consciousness may enhance moral status (Shepherd 2017, Koplin and Savulescu 2019b). Possessing self-consciousness would seem to give brain organoids a higher moral status than those with only phenomenal consciousness, as self-consciousness brings with 
FINAL DRAFT: 2021 in AJOB Neuroscience

it more critical needs and interests. Similarly, the potential for higher levels of distress can also endow brain organoids with a higher moral status (Koplin and Savulescu 2019b). However, the relationship between phenomenal consciousness and sophisticated cognitive abilities remains unclear. Phenomenal consciousness may or may not be necessary for such cognitive abilities (Niikawa 2018), for example. As it is not conceptually impossible for brain organoids to have such advanced cognitive abilities, the analysis of phenomenal consciousness alone would be unsatisfactory for the full ethical consideration.

\section{Ethical Issues raised by Transplanted Brain Organoids}

Recent years have seen some transplantations of human brain organoids into animal brains. If some would focus on the connection between the host and the graft via appropriate neural circuits in the transplantation studies, we would face the pressing need for ethical evaluation. Also, though too futuristic, this might soon be applied to therapeutic transplantation (Chen, et al. 2019a, 2019b). In the following section, we will present three ethical issues associated with transplantation studies, namely, (a) issues related to chimeras resulting from human brain organoid transplantation in animals, (b) issues of hybrids connected to non-living entities, and (c) issues associated with future transplantation into humans. We will also examine future directions for organoid transplantation research and application.

(a) Ethical issues related to the production of chimeric animals

The transplantation of more sophisticated human brain organoids into animals may produce chimeric animals with types of consciousness and cognitive abilities not seen in untransplanted animals. This possibility is the basis for concerns about the "moral humanization of animals," which can be differentiated from "biological humanization of animals": while both confer human-like properties to a particular animal, only the former does so in a way that carries moral significance. Humanization of animal hosts in both senses has been actively discussed in human-animal chimera research using the blastocyst 
FINAL DRAFT: 2021 in AJOB Neuroscience

complementation technique (see, for example, (Greely 2011, Hyun 2016, 2018)). However, the moral status implications of chimera studies which cause the moral humanization of animals, remains unclear (International Society for Stem Cell Research 2016, Koplin and Savulescu 2019a). With that, it is difficult to answer the ontological question of what features define the moral status of human-animal chimeras and the epistemological question of how competent animals are. Due to this moral uncertainty, some argue that animal rights should be taken into full consideration (Koplin and Wilkinson 2019).

Related to this, two ethical issues would be raised by transplanting human brain organoids in animal hosts: one is animal enhancement and human dignity that have been debated in the literature, and the other is significance of the subjective viewpoint that have been overlooked thus far. Conventional animal humanization concerns center on the dignity of humans and the enhancement of animals when animals acquire human cognitive or psychological/mental abilities (e.g. self-consciousness). Some argue that, if animals acquired psychological/mental abilities that only humans possess, this would violate human dignity, which would be morally problematic (Karpowicz, et al. 2005). As an example of animal disenhancement, in a research proposal about the intentional production of human-(non-ape) primate chimeras for modeling human neurological and psychiatric disease using blastocyst complementation, others show that the chimeric animals would be affected in ways that "compromise rather than enhance their normal capabilities and health" (De Los Angeles, et al. 2019). The other concern is that transplantation of brain organoids into the animal brains would change the brains of animal hosts in an unintended way. This might also raise some profound moral questions.

For the former, some argue that animal (dis)enhancement which does not give the animal self-consciousness is ethically acceptable when the benefit to humans outweighs the suffering of the animal (Savulescu 2011). Additionally, some authors argue that the creation of a human-animal chimera with psychological/mental ability that underlies human dignity constitutes the creation of new dignity, so that no one's (human) dignity is violated (Palacios-González 2015). In any case, the areas of brain organoid research which genuinely elicit concern about animal (dis)enhancement or violation of 
FINAL DRAFT: 2021 in AJOB Neuroscience

human dignity must be identified. We should judge the extent to which human brain organoids may permissibly be transplanted into animals for research use while taking into consideration animal enhancement and human dignity, the adequacy of experimental design and approach, and the welfare of involved animals (Hyun, et al. 2007, Porsdam Mann, et al. 2019). In the event that creating a chimeric animal with acquired selfconsciousness is deemed permissible, the resulting animal ought to be considered according to its moral status (Streiffer 2010, 2019).

For the latter, transplanting a brain organoid in vitro might raise the problem of unintended connection between brain organoids and the transplanted animal brain. There are two possible axes concerning the connection: (1) symmetric or asymmetric, and (2) phenomenal unity or compartment.

In symmetric cases, two autonomous, comprehensive minds will be connected. This may be the case between a matured-enough brain organoid in the future and an animal brain. However, we don't see an urgent reason to connect two mature brains, although some argue that this is a hypothetical possibility (Sotala and Valpola 2012). In contrast, in asymmetric cases, a brain organoid that realizes only partial psychological states will be transplanted. For example, this might include transplantation of a part of memory systems into a (malfunctioning) animal brain. Such an asymmetric connection would not raise the question whether one of the viewpoints would cease to exist, in so far as organoids with limited psychological capability would not instantiate phenomenal consciousness by themselves.

In comparison, cases of symmetric connection might entail either phenomenal unity or compartmentalization of consciousness. Phenomenal unity holds if and only if there is a single, total conscious state that encompasses (or subsumes) all the aspects of the subject's conscious experiences at a time (Bayne 2010). Our phenomenal consciousness is typically unified in this sense, although some argue that the consciousness of split-brain subjects might not (Schechter 2010). Phenomenal unity also guarantees only a single, unique subjective point of view within each organism. Hence, if symmetric connection were the case, the phenomenal unity scenario would eliminate one of the two unique perspectives. The possible disappearance of a subjective viewpoint 
FINAL DRAFT: 2021 in AJOB Neuroscience

due to such fusion may cast doubts on the moral permissibility of transplantation, even if the transplantation itself is initially permitted for some reasons. Though it is unclear whether such selves would also individuate moral units, which are demarcated from each other as targets of morally relevant behaviors, the subjective point of view might be a proper index of the number of moral units. Further discussion is needed on what defines the boundaries of moral units.

Phenomenal unity might not occur even with the symmetric connection case. In the compartmentalization of consciousness scenario, two subjective viewpoints would co-habit within one single organism, possibly interacting with each other. This might raise an ethical problem similar to conjoined twins (cf. (Savulescu and Persson 2016)), especially if two brains are irreversibly fused at the neural, tissue level.

The actual ethical concerns associated with these connections have yet to be fully clarified. To recap, the possibility of transplanting brain organoids into animal brains would force us to consider if such transplantation was morally permissible. Besides, we need to consider the fundamental moral problems, such as what it would mean morally to have a subjective perspective and lose such a viewpoint.

(b) Issues of hybrids connected to non-living entities

When discussing the moral importance of consciousness, we need to consider the potential consciousness of not only human brain organoids themselves or animals transplanted with human brain organoids, but also that of other hybrids connected to nonliving entities such as artificial intelligence (AI) and robots (Kahane and Savulescu 2009, Levy and Savulescu 2009). Some already argue that combining brain organoids in the future with other living or non-living systems may lead us to regard them as human individuals (Hyun, et al. 2020). Therefore, the moral status of brain organoids with "bodies" may need to be considered in conjunction with the moral status of the embryo or fetus. The possibility of brain organoids interacting with the external world or their surrounding environment by having a "body" raises the additional questions of what kinds of behavioral repertoires become possible and what moral implications these behaviors have. Collaboration with researchers in adjacent areas (such as AI) will become 
FINAL DRAFT: 2021 in AJOB Neuroscience

increasingly important in the future (Hyun, et al. 2020).

Some argue that phenomenal consciousness in a brain organoid ought not immediately lead to a ban on its experimental use (Koplin and Savulescu 2019b). However, even such philosophers admit that we should not use brain organoids with higher cognitive abilities for research purposes. Hence, if there is concern that connection of human brain organoids with non-living entities may induce higher cognitive abilities in hybrids, based on the precautionary principle, restrictions may potentially need to be imposed on such hybrid studies (Munthe 2019).

(c) Issues of future transplantation into human brains

In the future, it is expected that functional brain organoids will be transplanted to treat neurologic ailments including traumatic brain injury, and stroke. The possibility of therapeutic transplantation poses some ethical concerns. Similar to concerns relating to deep brain stimulation (DBS) (Gilbert, et al. 2018, Pugh, et al. 2018, Erler 2019), the transplantation of brain organoids to treat motor dysfunction could affect thoughts and behavior in an unintended or unpredictable way.

There is a significant difference between brain organoid transplantation and DBS, though, in the degree of reversibility of the two treatments. If DBS does not produce its desired therapeutic effect or if it produces an unwanted effect, the DBS electrode system can be turned on and off, and/or it can be removed altogether. It is believed that the effects and side effects of DBS treatment are reversed by removing DBS devices. In that sense, DBS is a reversible intervention.

Human brain organoids cannot be removed following transplantation, even if such a procedure does not have its desired outcome. It is possible that nerve cells from transplanted brain organoids may extend axons and create new neural pathways with the transplant recipient's own brain tissue, making the complete removal of the transplanted organoid difficult to impossible. In that sense, brain organoid transplantation is a highly invasive and irreversible intervention.

Other forms of therapeutic neural transplantation have previously been performed. Allogeneic transplantation using iPSC-derived dopaminergic neurons has 
FINAL DRAFT: 2021 in AJOB Neuroscience

been reported (Cyranoski 2018, Takahashi 2020). Brain organoids have more complex structures that may have cognitive function in their own right, thus raising the problem of psychological alteration due to transplanted brain organoids. Since this point cannot necessarily be resolved through animal experimentation alone, it may be an ethical obstacle in promoting clinical transplantation. On the other hand, even with this outstanding concern, medical scientists may try to use brain organoids for clinical application in patients with various brain-related diseases and their families may also desire clinical transplantation. Given these considerations, an appropriate ethical framework for clinical research based on proof of concept should be well established.

\section{Issues of Research Ethics and Governance}

Research using human-derived samples generally requires an ethically stringent process. For example, it is necessary to obtain appropriate informed consent from individuals who give samples and to ensure their right to withdraw consent during research in a transparent manner (World Medical Association 2013, Farahany, et al. 2018). Such consent is relevant to brain organoid research, as well. As brain organoids are produced from ESCs or iPSCs, a donor may give their cells as the seed of brain organoids, and in vitro brain organoids are a cloned entity of the donor. Nonetheless, they also have some types of consciousness, so that there may be cases where cell donors cannot withdraw consent because of organoids gaining consciousness. Thus, how to set the range of rights of the cell donor presents a problem in the production and use of brain organoids. Absent a clear answer, research ethics issues, i.e., the relations of cell donor to research materials or "research subjects" may arise.

It has been argued that brain organoid research poses the problem of stewardship and ownership -- if a brain organoid or an animal transplanted with the brain organoid acquires some form of consciousness which confers it moral status, who is responsible for the welfare of that brain organoid, and who owns it (Farahany, et al. 2018)? In the event of the creation of brain organoids or human-animal chimeras with selfconsciousness, their moral status should be equivalent to that of humans, and they ought to be treated in an appropriate manner. On this question, Chen et al. argue that, "[i]f there 
FINAL DRAFT: 2021 in AJOB Neuroscience

is evidence of rudimentary self-awareness, perhaps the chimeras should be removed from the research setting and retired to colonies such as chimpanzee sanctuaries. If there is further development of self-awareness, it may even be necessary to afford chimeras legal protections similar to humans, including consent for procedures and the right of selfdetermination" (Chen, et al. 2019b: 469). Before producing such chimeric animals as well as hybrids, we need to discuss how to grow and keep brain organoids, chimeras and hybrids, and how to consider the benefits and welfare of such entities who do not have verbal abilities (Savulescu 2011, Farahany, et al. 2018).

More practically, a further point of discussion involves which normative position to adopt for developing public policy. In the 1980s, the Warnock Committee in the UK discussed the ethical permissibility of human embryo research and adopted a utilitarian position that weighed costs and benefits to justify research use of human embryos (UK Department of Health and Social Security 1984: 65). In advocating for a utilitarian stance, Peter Singer argues that "[t]he capacity for suffering and enjoying things is a prerequisite for having interests at all, a condition that must be satisfied before we can speak of interests in any meaningful way... No matter what the nature of the being, the principle of equality requires that its suffering be counted equally with the like suffering - in so far as rough comparisons can be made - of any other being" (Singer 2011: 50). If sentience is a morally relevant property for brain organoid research, Singer's position dictates that the well-being of conscious beings (e.g. experimental animals) must be considered in planning and carrying out research. There are other, non-utilitarian moral frameworks that could inform policy debates. If the utilitarian position is not adopted, it is crucial that we decide which position will be taken up in its place.

In a different research context, He Jiankui's 2018 creation of twin girls using genome editing technology called CRISPR-Cas9 (Regalado 2018) has precipitated extensive ethical debate from academic groups and professional bodies about germline genome editing (National Academy of Sciences 2020). Currently, there is a broad consensus that reproductive uses of germline genome editing are unacceptable and irresponsible, though the question of a moratorium against such editing remains controversial (Lander, et al. 2019). One of the problems raised by germline genome 
FINAL DRAFT: 2021 in AJOB Neuroscience

editing is that undeveloped regulations and regulatory gaps between countries allow ethically gray research and clinical application loopholes. As international efforts to discuss ethical permissibility of emerging new technologies (e.g. germline genome editing) are underway (Jasanoff and Hurlbut 2018), the debate must include stakeholders in the production and use of brain organoids and set regulatory frameworks accordingly. As brain organoid research is closely related to other research domains such as AI and robotics, it is imperative to discuss the governance of organoid research in cooperation with adjacent areas of science and technology.

At the global level, articulating an international framework for research and application of brain organoids will be challenging, but undoubtedly necessary. The scientific community - including researchers, institutions, and funders - have an obligation to engage with the public and policy-makers about different moral positions and nuanced ethical challenges in research and application. National governments need to improve regulatory oversight to ensure that appropriate mechanisms are in place for the three categories of brain organoid, that is, in vitro brain organoid research, brain organoid transplantation in animals or brain organoid connection with non-living systems, and brain organoid transplantation in humans identified in the paper. Governance must all the while remain flexible, to accommodate the evolving science and be informed by the ethical considerations of individual societies. While it might be difficult to integrate different ethical norms and social and cultural concerns into a coherent instrument, developing international guidelines on scientific research practices, under the auspice of the World Health Organization, may be a starting point for international dialogue among governments, research institutes, and the public.

\section{Conclusion}

Following from the discussion up until this point, we propose three research policies for the ethics of brain organoid research and application.

Firstly, ethical issues should be identified and considered in anticipation of future research and clinical application of human brain organoids. With the rapid progress of 
FINAL DRAFT: 2021 in AJOB Neuroscience

brain organoid research, it is imperative to consider not only existing ethical challenges, but those likely to arise in the future. The path from basic to clinical application of human brain organoids has three phases of research: (i) in vitro studies of brain organoids, (ii) transplantation of human brain organoids into animal brains, and (iii) transplantation of human brain organoids into the human brain. As each phase involves different scientific and ethical issues, it would be useful to consider the phases separately.

The first phase of research will likely see further refinement of region-specific brain organoids (Benito-Kwiecinski and Lancaster 2020). This development will in turn facilitate the emergence of more complex brain organoids, created by fusing multiple region-specific brain organoids (Marton and Pașca 2020). This increased complexity may give such fused organoids capacities for sensory input and behavioral output. Capacity for sensory input and behavioral output should be a distinguishing characteristic in how we think about the consciousness of future brain organoids. Critically, as it is difficult to definitively determine whether brain organoids possess conscious experience, it may be advisable to adopt a precautionary approach that assumes future brain organoids will possess particular kinds of consciousness. (The authors of this paper have started this type of analysis).

In the second phase of research, transplanting immature human brain organoids into the brain of a living animal is not likely to lead to more sophisticated conscious experiences than the animal already possesses. Nevertheless, in the future, if a human brain organoid with more sophisticated brain structure and function is transplanted into an animal, the transplanted animal might develop higher brain function.

Secondly, to achieve the first policy, ethical evaluation needs to be well-informed by scientific research. To accurately estimate current and future research developments, there should be scientific input by researchers who conduct brain organoid research with expertise in related fields such as developmental biology and neuroscience. To prevent ethics and governance from being outpaced by scientific progress, scientists (including brain organoid researchers, but also biologists in neighboring fields and AI researchers), philosophers, legal scholars, and funding agencies should communicate with each other on both scientific facts and their resulting ethical, legal, and social implications. Also, 
FINAL DRAFT: 2021 in AJOB Neuroscience

alongside such interdisciplinary collaborations, it is also important to encourage public debates, involving a variety of stakeholders, on how to proceed with such research.

Thirdly, human brain organoid research and application both pose their own ethical and legal implications and exert a mutual influence on various other research areas. To ensure that impacts are both ethically and legally sound, it will be crucial to continue to develop human brain organoid research following appropriate guidelines established in each country as well as worldwide. It will be important for countries to develop appropriate regulations on human brain organoid research that are consistent with the ethical principles governing other types of biomedical research, e.g., research on embryos and non-human animals. In addition to national regulations, the development of an international regulatory framework is desirable, although this prospect presents familiar challenges, as illustrated for instance by the cases of human reproductive cloning or germline genome editing. However, such a framework may be necessary to ensure a minimal agreement exists at the international level over what is ethically and legally permissible in human brain organoid research.

\section{References}

Andersen, J., O. Revah, Y. Miura, N. Thom, N. D. Amin, K. W. Kelley, M. Signh, X. Chen, M. V. Thete, E. M. Walczak, H. Vogel, H. C. Fan, and S. Pașca. (2020). "Generation of Functional Human 3D Cortico-Motor Assembloids." Cell 20 31534-31538

Bayne, T. (2010). The Unity of Consciousness. Oxford, Oxford University Press.

Bayne, T. (2018). On the axiomatic foundations of the integrated information theory of consciousness. Neuroscience of consciousness. 2018(1): niy007.

Bayne, T., A. K. Seth and M. Massimini (2020). "Are There Islands of Awareness?" Trends Neurosci 43(1): 6-16.

Beauchamp, T. and D. DeGrazia (2020). Principles of Animal Research Ethics. Oxford, Oxford University Press.

Benito-Kwiecinski, S., and M. Lancaster. "Brain Organoids: Human Neurodevelopment 
FINAL DRAFT: 2021 in AJOB Neuroscience

in a Dish." Cold Spring Harb Perspect Biol 12(8): a035709.

Bhaduri, A., M. G. Andrews, W. Mancia Leon, D. Jung, D. Shin, D. Allen, D. Jung, G. Schmunk, M. Haeussler, J. Salma, A. A. Pollen, T. J. Nowakowski and A. R. Kriegstein (2020). "Cell stress in cortical organoids impairs molecular subtype specification." Nature 578(7793): 142-148.

Birch, J. (2017). "Animal sentience and the precautionary principle." Animal Sentience 16(1).

Boly, M., M. R. Coleman, M. H. Davis, A. Hampshire, D. Bor, G. Moonen, P. A. Maquet, J. D. Pickard, S. Laureys and A. M. Owen (2007). "When thoughts become action: an fMRI paradigm to study volitional brain activity in non-communicative brain injured patients." Neuroimage 36(3): 979-992.

Casali, A.G., Gosseries, O., Rosanova, M., Boly, M., Sarasso, S., Casali, K.R., Casarotto, S., Bruno, M.A., Laureys, S., Tononi, G. and Massimini, M., 2013. A theoretically based index of consciousness independent of sensory processing and behavior. Science Translational Medicine 5(198): 198ra105.

Chalmers, D. (1995). "Facing up to the problem of consciousness." Journal of Consciousness Studies 2: 200-219.

Chen, H. I., H. Song and G. L. Ming (2019a). "Applications of Human Brain Organoids to Clinical Problems." Dev Dyn 248(1): 53-64.

Chen, H. I., J. A. Wolf, R. Blue, M. M. Song, J. D. Moreno, G. L. Ming and H. Song (2019b). "Transplantation of Human Brain Organoids: Revisiting the Science and Ethics of Brain Chimeras." Cell Stem Cell 25(4): 462-472.

Cruse, D., S. Chennu, D. Fernandez-Espejo, W. L. Payne, G. B. Young and A. M. Owen (2012). "Detecting awareness in the vegetative state: electroencephalographic evidence for attempted movements to command." PLoS One 7(11): e49933.

Cugola, F. R., I. R. Fernandes, F. B. Russo, B. C. Freitas, J. L. Dias, K. P. Guimaraes, C. Benazzato, N. Almeida, G. C. Pignatari, S. Romero, C. M. Polonio, I. Cunha, C. L. Freitas, W. N. Brandao, C. Rossato, D. G. Andrade, P. Faria Dde, A. T. Garcez, C. A. Buchpigel, C. T. Braconi, E. Mendes, A. A. Sall, P. M. Zanotto, J. P. Peron, A. R. Muotri and P. C. Beltrao-Braga (2016). "The Brazilian Zika virus strain causes 
FINAL DRAFT: 2021 in AJOB Neuroscience

birth defects in experimental models." Nature 534(7606): 267-271.

Cyranoski, D. (2018). 'Reprogrammed' stem cells implanted into patient with Parkinson's disease. Nature.

Dang, J., S. K. Tiwari, G. Lichinchi, Y. Qin, V. S. Patil, A. M. Eroshkin and T. M. Rana (2016). "Zika Virus Depletes Neural Progenitors in Human Cerebral Organoids through Activation of the Innate Immune Receptor TLR3." Cell Stem Cell 19(2): 258-265.

Danjo, T., M. Eiraku, K. Muguruma, K. Watanabe, M. Kawada, Y. Yanagawa, J. L. Rubenstein and Y. Sasai (2011). "Subregional specification of embryonic stem cellderived ventral telencephalic tissues by timed and combinatory treatment with extrinsic signals." J Neurosci 31(5): 1919-1933.

Daviaud, N., R. H. Friedel and H. Zou (2018). "Vascularization and Engraftment of Transplanted Human Cerebral Organoids in Mouse Cortex." eNeuro 5(6).

Davies, W. and N. Levy (2016). Persistent vegetative state, akinetic mutism and consciousness. Finding Consciousness: The Neuroscience, Ethics, and Law of Severe Brain Damage. W. Sinnott-Armstrong. Oxford, Oxford University Press: 122-136.

De Los Angeles, A., I. Hyun, S. R. Latham, J. D. Elsworth and D. E. Redmond, Jr. (2019). "Human-Monkey Chimeras for Modeling Human Disease: Opportunities and Challenges." Methods Mol Biol 2005: 221-231.

Dehaene, S. and L. Naccache (2001). "Towards a cognitive neuroscience of consciousness: basic evidence and a workspace framework." Cognition 79(1-2): 137.

Eiraku, M., N. Takata, H. Ishibashi, M. Kawada, E. Sakakura, S. Okuda, K. Sekiguchi, T. Adachi and Y. Sasai (2011). "Self-organizing optic-cup morphogenesis in threedimensional culture." Nature 472(7341): 51-56.

Eiraku, M., K. Watanabe, M. Matsuo-Takasaki, M. Kawada, S. Yonemura, M. Matsumura, T. Wataya, A. Nishiyama, K. Muguruma and Y. Sasai (2008). "Self-organized formation of polarized cortical tissues from ESCs and its active manipulation by extrinsic signals." Cell Stem Cell 3(5): 519-532. 
FINAL DRAFT: 2021 in AJOB Neuroscience

Erler, A. (2019). "Discussions of DBS in neuroethics: Can we deflate the bubble without deflating ethics?" Neuroethics.

Farahany, N. A., H. T. Greely, S. Hyman, C. Koch, C. Grady, S. P. Pasca, N. Sestan, P. Arlotta, J. L. Bernat, J. Ting, J. E. Lunshof, E. P. R. Iyer, I. Hyun, B. H. Capestany, G. M. Church, H. Huang and H. Song (2018). "The ethics of experimenting with human brain tissue." Nature 556(7702): 429-432.

Fernandez-Espejo, D. and A. M. Owen (2013). "Detecting awareness after severe brain injury." Nat Rev Neurosci 14(11): 801-809.

Garcez, P. P., E. C. Loiola, R. Madeiro da Costa, L. M. Higa, P. Trindade, R. Delvecchio, J. M. Nascimento, R. Brindeiro, A. Tanuri and S. K. Rehen (2016). "Zika virus impairs growth in human neurospheres and brain organoids." Science 352(6287): 816-818.

Gilbert, F., J. N. M. Viaña and C. Ineichen (2018). "Deflating the "DBS causes personality changes" bubble." Neuroethics.

Greely, H. T. (2011). Human/nonhuman chimeras: Assessing the issues. The Oxford Handbook of Animal Ethics. T. Beauchamp and R. G. Frey. Oxford, Oxford University Press: 671-698.

Hasegawa, Y., N. Takata, S. Okuda, M. Kawada, M. Eiraku and Y. Sasai (2016). "Emergence of dorsal-ventral polarity in ESC-derived retinal tissue." Development 143(21): 3895-3906.

Hostiuc, S., M. C. Rusu, I. Negoi, P. Perlea, B. Dorobantu and E. Drima (2019). "The moral status of cerebral organoids." Regen Ther 10: 118-122.

Hyun, I. (2016). "What's Wrong with Human/Nonhuman Chimera Research?" PLoS Biol 14(8): e1002535.

Hyun, I. (2018). "The Ethics of Chimera Creation in Stem Cell Research." Current Stem Cell Reports 4(3): 235-239.

Hyun, I., J. C. Scharf-Deering and J. E. Lunshof (2020). "Ethical issues related to brain organoid research." Brain Res 1732: 146653.

Hyun, I., P. Taylor, G. Testa, B. Dickens, K. W. Jung, A. McNab, J. Robertson, L. Skene and L. Zoloth (2007). "Ethical standards for human-to-animal chimera experiments 
FINAL DRAFT: 2021 in AJOB Neuroscience

in stem cell research." Cell Stem Cell 1(2): 159-163.

Ishida, Y., H. Kawakami, H. Kitajima, A. Nishiyama, Y. Sasai, H. Inoue and K. Muguruma (2016). "Vulnerability of Purkinje Cells Generated from Spinocerebellar Ataxia Type 6 Patient-Derived iPSCs." Cell Rep 17(6): 1482-1490.

Jasanoff, S. and J. B. Hurlbut (2018). "A global observatory for gene editing." Nature 555(7697): 435-437.

Kadoshima, T., H. Sakaguchi, T. Nakano, M. Soen, S. Ando, M. Eiraku and Y. Sasai (2013). "Self-organization of axial polarity, inside-out layer pattern, and speciesspecific progenitor dynamics in human ES cell-derived neocortex." Proc Natl Acad Sci U S A 110(50): 20284-20289.

Kahane, G. and J. Savulescu (2009). "Brain damage and the moral significance of consciousness." J Med Philos 34(1): 6-26.

Karpowicz, P., C. B. Cohen and D. van der Kooy (2005). "Developing human-nonhuman chimeras in human stem cell research: ethical issues and boundaries." Kennedy Inst Ethics $J$ 15(2): 107-134.

Kitahara, T., H. Sakaguchi, A. Morizane, T. Kikuchi, S. Miyamoto and J. Takahashi (2020). "Axonal Extensions along Corticospinal Tracts from Transplanted Human Cerebral Organoids." Stem Cell Reports 15(2): 467-481.

Koplin, J. and D. Wilkinson (2019). "Moral uncertainty and the farming of human-pig chimeras." J Med Ethics 45(7): 440-446.

Koplin, J. J. and J. Savulescu (2019a). "Time to rethink the law on part-human chimeras." J Law Biosci 6(1): 37-50.

Koplin, J. J. and J. Savulescu (2019b). "Moral Limits of Brain Organoid Research." J Law Med Ethics 47(4): 760-767.

Kuwahara, A., C. Ozone, T. Nakano, K. Saito, M. Eiraku and Y. Sasai (2015). "Generation of a ciliary margin-like stem cell niche from self-organizing human retinal tissue." Nat Commun 6: 6286.

Lancaster, M. A., M. Renner, C. A. Martin, D. Wenzel, L. S. Bicknell, M. E. Hurles, T. Homfray, J. M. Penninger, A. P. Jackson and J. A. Knoblich (2013). "Cerebral organoids model human brain development and microcephaly." Nature 501(7467): 
FINAL DRAFT: 2021 in AJOB Neuroscience

373-379.

Lander, E. S., F. Baylis, F. Zhang, E. Charpentier, P. Berg, C. Bourgain, B. Friedrich, J. K. Joung, J. Li, D. Liu, L. Naldini, J. B. Nie, R. Qiu, B. Schoene-Seifert, F. Shao, S. Terry, W. Wei and E. L. Winnacker (2019). "Adopt a moratorium on heritable genome editing." Nature 567(7747): 165-168.

Lavazza, A. (2020). "Human cerebral organoids and consciousness: a double-edged sword." Monash Bioeth Rev.

Lavazza, A. and M. Massimini (2018). "Cerebral organoids: ethical issues and consciousness assessment." J Med Ethics 44(9): 606-610.

Lee, A. (2019). "Is consciousness intrinsically valuable?" Philosophical Studies 175(1): $1-17$.

Levy, N. and J. Savulescu (2009). "Moral significance of phenomenal consciousness." Prog Brain Res 177: 361-370.

Madhavan, A., G. D. Carnaby, K. Chhabria and M. A. Crary (2018). "Preliminary Development of a Screening Tool for Pre-Clinical Dysphagia in Community Dwelling Older Adults." Geriatrics (Basel) 3(4).

Mansour, A. A., Gonçalves, J. T., Bloyd, C. W., Li, H., Fernandes, S., Quang, D., Johnston, S., Parylak, S. L., Jin, X., and Gage, F. H. (2018). An in vivo model of functional and vascularized human brain organoids. Nat Biotechnol, 36(5), 432-441. doi:10.1038/nbt.4127

Marton, R.M., and S. Pașca. (2020). "Organoid and Assembloid Technologies for Investigating Cellular Crosstalk in Human Brain Development and Disease.” Trends Cell Biol 30(2): 133-143.

Monti, M. M., A. Vanhaudenhuyse, M. R. Coleman, M. Boly, J. D. Pickard, L. Tshibanda, A. M. Owen and S. Laureys (2010). "Willful modulation of brain activity in disorders of consciousness." N Engl J Med 362(7): 579-589.

Muguruma, K., A. Nishiyama, H. Kawakami, K. Hashimoto and Y. Sasai (2015). "Selforganization of polarized cerebellar tissue in $3 \mathrm{D}$ culture of human pluripotent stem cells." Cell Rep 10(4): 537-550.

Munsie, M., I. Hyun and J. Sugarman (2017). "Ethical issues in human organoid and 
FINAL DRAFT: 2021 in AJOB Neuroscience

gastruloid research." Development 144(6): 942-945.

Munthe, C. (2019). "Price of precaution of human-pig chimeras for transplantation purposes." J Med Ethics 45(7): 447-448.

Nagel, T. (1974). "What is it like to be a bat?" The Philosophical Review 83(4): 435-450.

Nakano, T., S. Ando, N. Takata, M. Kawada, K. Muguruma, K. Sekiguchi, K. Saito, S. Yonemura, M. Eiraku and Y. Sasai (2012). "Self-formation of optic cups and storable stratified neural retina from human ESCs." Cell Stem Cell 10(6): 771-785.

Nasu, M., N. Takata, T. Danjo, H. Sakaguchi, T. Kadoshima, S. Futaki, K. Sekiguchi, M. Eiraku and Y. Sasai (2012). "Robust formation and maintenance of continuous stratified cortical neuroepithelium by laminin-containing matrix in mouse ES cell culture." PLoS One 7(12): e53024.

National Academy of Sciences. (2020). Heritable Human Genome Editing. Washington, DC: The National Academies Press.

Niikawa, T. (2018). "Moral status and consciousness " The Annals of the University of Bucharest - Philosophy series 67(1): 235-257.

Ormel, P. R., R. Vieira de Sa, E. J. van Bodegraven, H. Karst, O. Harschnitz, M. A. M. Sneeboer, L. E. Johansen, R. E. van Dijk, N. Scheefhals, A. Berdenis van Berlekom, E. Ribes Martinez, S. Kling, H. D. MacGillavry, L. H. van den Berg, R. S. Kahn, E. M. Hol, L. D. de Witte and R. J. Pasterkamp (2018). "Microglia innately develop within cerebral organoids." Nat Commun 9(1): 4167.

Owen, A. M. (2019). "The search for consciousness." Neuron 102(3): 526-528.

Owen, A. M., M. R. Coleman, M. Boly, M. H. Davis, S. Laureys and J. D. Pickard (2006). "Detecting awareness in the vegetative state." Science 313(5792): 1402.

Ozone, C., H. Suga, M. Eiraku, T. Kadoshima, S. Yonemura, N. Takata, Y. Oiso, T. Tsuji and Y. Sasai (2016). "Functional anterior pituitary generated in self-organizing culture of human embryonic stem cells." Nat Commun 7: 10351.

Palacios-Gonzalez, C. (2015). "Human dignity and the creation of human-nonhuman chimeras." Med Health Care Philos 18(4): 487-499.

Pollen, A. A., A. Bhaduri, M. G. Andrews, T. J. Nowakowski, O. S. Meyerson, M. A. Mostajo-Radji, E. Di Lullo, B. Alvarado, M. Bedolli, M. L. Dougherty, I. T. Fiddes, 
FINAL DRAFT: 2021 in AJOB Neuroscience

Z. N. Kronenberg, J. Shuga, A. A. Leyrat, J. A. West, M. Bershteyn, C. B. Lowe, B. J. Pavlovic, S. R. Salama, D. Haussler, E. E. Eichler and A. R. Kriegstein (2019). "Establishing Cerebral Organoids as Models of Human-Specific Brain Evolution." Cell 176(4): 743-756 e 717.

Porsdam Mann, S., R. Sun and G. Hermeren (2019). "A framework for the ethical assessment of chimeric animal research involving human neural tissue." BMC Med Ethics 20(1): 10.

Pugh, J., L. Pycroft, H. Maslen, T. Aziz and J. Savulescu (2018). "Evidence-Based Neuroethics, Deep Brain Stimulation and Personality - Deflating, but not Bursting, the Bubble." Neuroethics.

Qian, H., X. Kang, J. Hu, D. Zhang, Z. Liang, F. Meng, X. Zhang, Y. Xue, R. Maimon, S. F. Dowdy, N. K. Devaraj, Z. Zhou, W. C. Mobley, D. W. Cleveland and X. D. Fu (2020). "Reversing a model of Parkinson's disease with in situ converted nigral neurons." Nature 582(7813): 550-556.

Qian, X., H. N. Nguyen, M. M. Song, C. Hadiono, S. C. Ogden, C. Hammack, B. Yao, G. R. Hamersky, F. Jacob, C. Zhong, K. J. Yoon, W. Jeang, L. Lin, Y. Li, J. Thakor, D. A. Berg, C. Zhang, E. Kang, M. Chickering, D. Nauen, C. Y. Ho, Z. Wen, K. M. Christian, P. Y. Shi, B. J. Maher, H. Wu, P. Jin, H. Tang, H. Song and G. L. Ming (2016). "Brain-Region-Specific Organoids Using Mini-bioreactors for Modeling ZIKV Exposure." Cell 165(5): 1238-1254.

Quadrato, G., T. Nguyen, E. Z. Macosko, J. L. Sherwood, S. Min Yang, D. R. Berger, N. Maria, J. Scholvin, M. Goldman, J. P. Kinney, E. S. Boyden, J. W. Lichtman, Z. M. Williams, S. A. McCarroll and P. Arlotta (2017). "Cell diversity and network dynamics in photosensitive human brain organoids." Nature 545(7652): 48-53.

Regalado, A. (2018). EXCLUSIVE: Chinese scientists are creating CRISPR babies. MIT Technology Review.

Research, I. S. f. S. C. (2016). Guidelines for Stem Cell Research and Clinical Translation. Sakaguchi, H., T. Kadoshima, M. Soen, N. Narii, Y. Ishida, M. Ohgushi, J. Takahashi, M. Eiraku and Y. Sasai (2015). "Generation of functional hippocampal neurons from self-organizing human embryonic stem cell-derived dorsomedial telencephalic 
FINAL DRAFT: 2021 in AJOB Neuroscience

tissue." Nat Commun 6: 8896.

Sakaguchi, H., Y. Ozaki, T. Ashida, T. Matsubara, N. Oishi, S. Kihara and J. Takahashi (2019). "Self-Organized Synchronous Calcium Transients in a Cultured Human Neural Network Derived from Cerebral Organoids." Stem Cell Reports 13(3): 458473.

Samarasinghe, R. A., O. A. Miranda, S. Mitchell, I. Ferando, M. Watanabe, J. E. Buth, A. Kurdian, P. Golshani, K. Plath, W. E. Lowry, J. M. Parent, I. Mody and B. G. Novitch (2019). "Identification of neural oscillations and epileptiform changes in human brain organoids." bioRxiv $\mathbf{8 2 0 1 8 3 .}$

Savulescu, J. (2011). Genetically modified animals: Should there be limits to engineering the animal kingdom? The Oxford Handbook of Animal Ethics. T. Beauchamp and R. G. Frey. Oxford, Oxford University Press.

Savulescu, J. and I. Persson (2016). "Conjoined Twins: Philosophical Problems and Ethical Challenges." J Med Philos 41(1): 41-55.

Sawai, T., H. Sakaguchi, E. Thomas, J. Takahashi and M. Fujita (2019). "The Ethics of Cerebral Organoid Research: Being Conscious of Consciousness." Stem Cell Reports 13(3): 440-447.

Schechter, E. (2010). "Individuating mental tokens: The split-brain case." Philosophia 38(1): 195-216.

Shepherd, J. (2017). "The Moral Insignificance of Self-consciousness." Eur J Philos 25(2): 398-415.

Shepherd, J. (2018). Consciousness and Moral Status. Oxford, Routledge.

Shepherd, J. (2018). "Ethical (and epistemological) issues regarding consciousness in cerebral organoids." J Med Ethics 44(9): 611-612.

Shepherd, J. (forthcoming). "The moral status of conscious subjects." Rethinking Moral Status. ed. S. Clarke, H. Zohny, and J. Savulescu. Oxford: Oxford University Press.

Shiraishi, A., K. Muguruma and Y. Sasai (2017). "Generation of thalamic neurons from mouse embryonic stem cells." Development 144(7): 1211-1220.

Singer, P. (2011). Practical Ethics. NY, Cambridge University Press.

Sotala, K. A. J. and H. Valpola (2012). "Coalescing Minds: Brain Uploading-Related 
FINAL DRAFT: 2021 in AJOB Neuroscience

Group Mind Scenarios." International Journal of Machine Consciousness 04(01): 293-312.

Streiffer, R. (2010). "Chimeras, moral status, and public policy: implications of the abortion debate for public policy on human/nonhuman chimera research." J Law Med Ethics 38(2): 238-250.

Streiffer, R. (2019). Human/Non-Human Chimeras. The Stanford Encyclopedia of Philosophy. E. Zalta.

Suga, H., T. Kadoshima, M. Minaguchi, M. Ohgushi, M. Soen, T. Nakano, N. Takata, T. Wataya, K. Muguruma, H. Miyoshi, S. Yonemura, Y. Oiso and Y. Sasai (2011). "Self-formation of functional adenohypophysis in three-dimensional culture." Nature 480(7375): 57-62.

Takahashi, J. (2020). "Preclinical evaluation of patient-derived cells shows promise for Parkinson's disease." J Clin Invest 130(2): 601-603.

Takata, N., E. Sakakura, M. Eiraku, T. Kasukawa and Y. Sasai (2017). "Self-patterning of rostral-caudal neuroectoderm requires dual role of Fgf signaling for localized Wnt antagonism." Nat Commun 8(1): 1339.

Tononi, G. (2012). "Integrated information theory of consciousness: an updated account." Arch Ital Biol 150(4): 293-329.

Tononi, G., M. Boly, M. Massimini and C. Koch (2016). "Integrated information theory: from consciousness to its physical substrate." Nat Rev Neurosci 17(7): 450-461.

Trujillo, C. A., R. Gao, P. D. Negraes, J. Gu, J. Buchanan, S. Preissl, A. Wang, W. Wu, G. G. Haddad, I. A. Chaim, A. Domissy, M. Vandenberghe, A. Devor, G. W. Yeo, B. Voytek and A. R. Muotri (2019). "Complex Oscillatory Waves Emerging from Cortical Organoids Model Early Human Brain Network Development." Cell Stem Cell 25(4): 558-569 e557.

UK Department of Health \& Social Security. (1984). Report of the committee of inquiry into human fertilisation and embryology. London: Her Majesty's Stationary Office.

Van Gulick, R. (2018). Consciousness. The Stanford Encyclopedia of Philosophy. E. Zalta.

Visak, T. and Garner R. (2016). The Ethics of Killing Animals. Oxford, Oxford University Press. 
FINAL DRAFT: 2021 in AJOB Neuroscience

Watanabe, M., J. E. Buth, N. Vishlaghi, L. de la Torre-Ubieta, J. Taxidis, B. S. Khakh, G. Coppola, C. A. Pearson, K. Yamauchi, D. Gong, X. Dai, R. Damoiseaux, R. Aliyari, S. Liebscher, K. Schenke-Layland, C. Caneda, E. J. Huang, Y. Zhang, G. Cheng, D. H. Geschwind, P. Golshani, R. Sun and B. G. Novitch (2017). "Self-Organized Cerebral Organoids with Human-Specific Features Predict Effective Drugs to Combat Zika Virus Infection." Cell Rep 21(2): 517-532.

Watanabe, M., Y. J. Kang, L. M. Davies, S. Meghpara, K. Lau, C. Y. Chung, J. Kathiriya, A. K. Hadjantonakis and E. S. Monuki (2012). "BMP4 sufficiency to induce choroid plexus epithelial fate from embryonic stem cell-derived neuroepithelial progenitors." J Neurosci 32(45): 15934-15945.

Wataya, T., S. Ando, K. Muguruma, H. Ikeda, K. Watanabe, M. Eiraku, M. Kawada, J. Takahashi, N. Hashimoto and Y. Sasai (2008). "Minimization of exogenous signals in ES cell culture induces rostral hypothalamic differentiation." Proc Natl Acad Sci U S A 105(33): 11796-11801.

Wiese, J. (2020). "The science of consciousness does not need another theory, it needs a minimal unifying model." Neuroscience of Consciousness 6(1): niaa013.

World Medical Association. (2013). "World Medical Association Declaration of Helsinki: ethical principles for medical research involving human subjects." JAMA 310(20): 2191-2194.

Xiang, Y., Y. Tanaka, B. Cakir, B. Patterson, K. Y. Kim, P. Sun, Y. J. Kang, M. Zhong, X. Liu, P. Patra, S. H. Lee, S. M. Weissman and I. H. Park (2019). "hESC-Derived Thalamic Organoids Form Reciprocal Projections When Fused with Cortical Organoids." Cell Stem Cell 24(3): 487-497 e487. 